\title{
Integrating Service Design and Eye Tracking Insight for Designing Smart TV User Interfaces
}

\author{
Sheng-Ming Wang \\ Department of Interaction Design, \\ National Taipei University of Technology, \\ Taipei, TAIWAN
}

\begin{abstract}
This research proposes a process that integrate service design method and eye tracking insight for designing a Smart TV user interface. The Service Design method, which is utilized for leading the combination of the quality function deployment (QFD) and the analytic hierarchy process (AHP), is used to analyze the features of three Smart TV user interface design mockups. Scientific evidences, which include the effectiveness and efficiency testing data obtained from eye tracking experiments with six participants, are provided the information for analysing the affordance of these design mockups. The results of this research demonstrate a comprehensive methodology that can be used iteratively for redesigning, redefining and evaluating of Smart TV user interfaces. It can also help to make the design of Smart TV user interfaces relate to users' behaviors and needs. So that to improve the affordance of design. Future studies may analyse the data that are derived from eye tracking experiments to improve our understanding of the spatial relationship between designed elements in a Smart TV user interface.
\end{abstract}

Keywords-Smart TV; User Interface Design; Eye Tracking; Design Affordance; Service Design

\section{INTRODUCTION}

Smart TV devices provide both broadcast and broadband content on a TV $[1,2]$. They facilitate the curation of content by combining Internet-based information with content from TV providers[3]. These devices have the potential to combine the strengths of TV broadcasting and broadband network services. Accordingly, Smart TV depends innovative human-computer interaction(HCIs) to provide suitable services and to meet user requirements $[4,5]$. Unlike those of a conventional TV with a remote control, new Smart TV features, such as web search, social networking, multi-user operation, personalized services and application development, require innovative "natural" HCIs[6]. Not only are the interactions and functions of a Smart TV user interface(UI) important, but also is its adaptability to individual users. To develop a Smart TV UI with service, researchers with technical and design backgrounds must work together in an interdisciplinary fashion with a comprehensive roadmap that specifies relevant requirements [3, 4, 7]. Although a Smart TV serves audiences by the delivery of innovative services, a number of questions about the mechanism of that delivery to various users via a single platform remain. Smart TV HCIs and UIs are important to all users, content providers and Smart TV manufacturers because innovative HCIs and UIs are required to provide enhanced services and meet user requirements[4, 8]. Numerous works have established the importance of different criteria in designing HCIs and UIs[9-13]. Some researchers have proposed various processes for designing Smart TV HCIs and UIs [3, 5, 13, 14]. However, evaluation of the effectiveness, efficiency, and usability or, more generally, the affordance, of Smart TV HCI and UI designs results requires further investigation. Therefore, this work develops a comprehensive methodology for evaluating the design affordance of Smart TV UIs. Affordance evaluation is a technique that is widely used to identify the quality of various aspects of web site design[15, 16], product design[17], interaction design[18], and engineering design[19]. The purpose of an affordance evaluation is to ensure that users of the Smart TV UIs can use design mockups efficiently and effectively. The two classes of affordance evaluation are empirical methods and inspections. Empirical methods are based on observing, capturing, and analyzing data about usage by real end-users, while inspections are conducted by expert evaluators or designers, and involve reviewing the usability-related aspects of the design(such as mock-ups, conceptual models, user interfaces), commonly associated with UIs, with regard to their conformance with a set of guidelines. This research mostly concerns inspections. Firstly, in an interdisciplinary service design workshop, Quality Function Deployment (QFD) and the Analytic Hierarchy Process (AHP) were integrated to derive qualitative and quantitative information about user-related scenarios and user requirements for developing three Smart TV UI mockup designs. Then, eye tracking evaluations are performed with six participants to gather gazing data, eye paths, and heat maps for further analysis. The results of the eye tracking analysis are then compared with the results obtained from a service design workshop to evaluate the design affordance of the Smart TV UIs.

The remainder of this paper is organized as follows. Section II reviews related work. Section III discusses the relevant design mechanism and proposed methodology for Smart TV UIs design. Section IV discusses results of the implementation of QFD and AHP for a Smart TV UI design mockup. Section $\mathrm{V}$ presents the eye tracking results and analyzes the Smart TV UI design affordance. Section V draws conclusions and offers idea for future works.

\section{RELATED WORKS}

\section{A. Services design method for develpoing smart tv uis}

Service interfaces are designed for intangible products that are, from the customer's point of view, useful, profitable and 
desirable, while they are effective, efficient and different for the provider. The method for making this process integral and holistic is to incorporate the particular visions of all stakeholders, including users, designers, investors, researchers, technicians, policy makers, consultants and competitors. Bill Moggridge offered the following definition; "service design is the design of intangible experiences that reach people through many different touch-points"[11]. That is, service design is a process of continual updates based on the responses of users who are observed and monitored. Service design is a means of transferring traditional product design and interface design to commercial services. Also, service design can help to elucidate user requirements and find solutions to the design of services, products, and other related elements to users. The principles of service design have been implemented in scenario planning[11, 22].

By integrating the internet into television sets, Smart TVs allow consumers to use on-demand streaming media services, listen to radio, access interactive media, use social networks, and download applications[20]. Nowadays, Smart TVs not only offer access to the internet and legacy web services, but also provide content services that are immediately coupled to broadcast content that is rendered on the terminal device[2]. To provide more and better services, a Smart TV must have a menu system and UI that can be navigated to complete a task. As several researched have noted, an intuitive and easily navigated HCI and UI are critical to a good user experience of a Smart TV[1, 9, 13, 18, 21]. Hence, a comprehensive process that includes design and evaluation of Smart TV UIs is very important for making Smart TV services more desirable and useful .Unlike the features of a conventional TV with a remote control, new Smart TV features, such as web search, social networking, multi-user support, personalized services and application development, depend on innovative "natural" HCIs[4]. To improve the HCIs with a Smart TV, our earlier work brought together technicians and designers in an inter-disciplinary context to generate a comprehensive roadmap for the development of Smart TVs and identify future requirements thereof $[3,5]$. Any application of service design to the multimodal interaction development of Smart TV must consider aspects of both product design and interface design. Moreover, this work follows some features and characteristics of service design that were summarized as follows.

\section{1) Assessing services from a holistic and detailed point of} view.

2) Considering both artifacts and experiences.

3) Making services tangible and visible via visualizations.

\section{B. QFD-AHP Integration for Smart TV UIs Design}

In addition to the service design approach, the quality function deployment(QFD) matrix and analytical hierarchy process(AHP) method are also utilized simultaneously to systematically identify the criteria derived from service design scenario planning, and to weight and prioritize criteria.

The (QFD) method is a qualitative approach that is used to systematically assess the correlation between user requirements and technical features. The QFD matrix is a systematic design approach based on an in-depth awareness of customer desires, coupled with integrated corporate functional groups. The QFD matrix translates customer desires into design characteristics for each stage of product development. The ultimate goal of is to translate often subjective criteria into objective criteria that can be quantified and measured and which can then be used to design and manufacture the product. However, it has two weaknesses: firstly, it does not prioritize customer requirements; secondly, the weights are subjectively evaluated and depend on consensus among a panel of experts.

The AHP method is a structured technique that converge the opinions from domain experts for dealing with complex decisions[23, 24]. The AHP enables groups of people to interact and focus on a certain problem, modify their judgments and, as a result, combine group judgments in accordance with the main criteria. Applying the AHP to weight CRs in a QFD matrix provides a rational framework for structuring a decision problem. The combined AHP-QFD approach can quantify CRs and elements, relate those elements to overall $\mathrm{CR}$ goals and evaluate alternative solutions. The combined AHP-QFD approach has been used successfully to assess customer needs based on a multiple-choice decision analysis. Gupta et al. reviewed uses of the QFD-AHP to evaluate and select methodology for an innovative product design concept. The methodology combining QFD-AHP was mainly used as a multi-criteria decision method for evaluating user requirements. By considering the requirements of designing Smart TVs and characteristics of service design, this work uses this methodology to evaluate the design and development of Smart TV UIs.

\section{Evaluation of Design Affordance and Eye-Tracking}

The perceptual psychologist J. J. Gibson coined the word "affordance" to refer to the actionable properties between the world and an actor[26]. Don Norman argues that, to Gibson, affordances are relationships. They exist naturally: they do not have to be visible, known, or desirable[27] ; he would make a global change, replacing all instances of the word "affordance" with the expression "perceived affordance." Don Norman also points out that affordances, both real and perceived, play very different roles in physical products, from their roles in screen-based products, such as UIs design. In product design, which deals with real, physical objects, both real and perceived affordances are involved, and the two sets may differ. With respect to graphical, screen-based interfaces, a designer primarily can control only perceived affordances[27]. Obrenovic and Starcevic claimed that HCIs are moving the balance of interactions closer to the human and support expressive, transparent, efficient, and robust interaction[10]. Therefore, the design of Smart TV UIs should focus on usability, which refers to "the extent to which a product can be used by specified users to achieve specified goals with effectiveness, efficiency and satisfaction in a specified context of use."[9] However, usability issues commonly arise concerning HCI and UI navigation that has been poorly designed, typically because of the organization, placement, visual design, or terminology involved. Current methods for measuring the effectiveness of navigation are limited to observable behaviors and verbal feedback from participants.

Eye tracking is becoming an increasingly common tool in UX testing, enabling new ways to optimize navigational elements in UI design to be discovered [9]. Research into 
results for evaluating eye tracking indicates that the relationship between interface and human reading habits can be recorded and analyzed systematically and scientifically to provide references for UI design[28, 29]. A book entitled "Measuring the User Experience: Collecting, Analyzing, and Presenting", states that Eye Tracking (Gaze Tracker) is a good example of evaluating user experience and does not measuring not only where an eye is looking but also its motion, which makes measurement more easier and accurate[30]. A group of researchers used Gaze Tracker to determine whether object placement based on user expectation results in faster and better recall of the object's location[31]. Aesthetic usability effect is influenced by the user's affective response to the interaction, so Smart TV UI design should be evaluated to determine whether users recognize something that they appreciate and find to be aesthetically pleasing. Another issue proposed by Soussan Djamasbi found that Gaze Tracker can be utilized to measure the preferences of members of Generation Y (age 18-31) among various interfaces[29]. This research addresses how eye tracking can be used to understand the effectiveness of the design of mockups of Smart TV UIs, based on information derived from the QFD-AHP model. Three interface mockups were displays on a screen and Gaze Tracker was used to collect the gaze data of participants for 20 minutes. The evaluation of the eye tracking function was then used in the roadmap for designing Smart TV UIs to review the results.

\section{MethodOLOGY}

\section{A. Define the Features of Smart TV UIS}

The concept of design thinking is integrated here with technology development to develop a Smart TV UI. The four major phases of the interdisciplinary integration of the design thinking concept with technology development are as follows. The first phase is to outline the vision for the Smart TV HCI design, based on a review of trends of the development of, and visions for, Smart TVs. The second phase is to propose a plan for the interdisciplinary integration of technology and experts in the domain of interaction design by holding service design workshops to foster interdisciplinary brainstorming. The third phase, which provides the kernel of the integration, is to define user scenarios and technology benchmarks based on insights into user-centered design. In the last phase, the combined QFD-AHP approach is implemented to analyze the features and classes of Smart TV UIs mockup designs. Finally, an eye-tracking evaluation is conducted to the pupil is tracked and its movement is recorded for further analysis. The results are used to evaluate the design affordance of the developed Smart TV UIs.

\section{B. QFD-AHP Model}

The QFD matrix specifies the importance of each feature based on a correlation analysis of user requirements and technical features. It also shows user recognition by describing their experiences to competitors by giving a value to their importance. The importance range is $1-5$ and their thinking is limited to strong, moderate, or poor. This method reveals how strongly the features (product characteristics) are related to user requirements and reflects the strengths of existing products. This work uses the QFD matrix to systematically list the features of the multimodal HCI design. This work uses the
QFD matrix to list systematically the features of Smart TV UI design. An inter-disciplinary team of faculty members, researchers and graduate students with interaction design, computer science, and electronic engineering backgrounds participated in a service design workshop. This workshop helped the team gain a clear understanding of the features of Smart TV UIs design. The many ideas of the Smart TV UIs design that were generated in the workshop were narrowed down from global thoughts to specific and applicable features that meet user requirements and could be developed technically. All of the features that were obtained from service design workshop are represented in a QFD matrix. The importance of each feature is obtained by performing a correlation analysis of user requirements and the technical features that users demand.

After the QFD analysis, the AHP method is used to evaluate the results. The three basic steps in the AHP research areas follows.

1) Describe a complex decision-making problem as a hierarchy.

2) Perform pair wise comparison to estimate the priorities of various elements on each level of the hierarchy.

3) Integrate these priorities to obtain an overall evaluation of decision alternatives.

The AHP calculation template that was provided by Goepel[32] is used here for the primitive analysis of AHP results. The result workbook consists of 20 input worksheets for pair wise comparisons, a sheet consolidating all assessments, a summary sheet of systematic results, a sheet of reference tables ( a random index, limits for the geometric consistency index (GCI), and judgment scales) and a sheet for solving the eigenvalue problem using the eigenvector method (EVM).

\section{Eye Tracking Evaluation}

A gaze tracking device, EyeLink 1000 Plus, which was made available by the National Taipei University of Technology, was used to trace participants' eye movements. The device was set $2.75 \mathrm{~m}$ away from optical receiver. Since the device comes an infrared lamp, the room was made dark to eliminate any inaccuracy during the experiment. The evaluation involved six participants, who were asked to sync their eye's gaze to the sensor before beginning the task. Participants were required to describe the contents of a selected Smart TV UI design, while the Gaze Tracker recorded their eye position. The obtained were presented as eye movement points and timed trajectories. GazeTrail, LookZone, and HeatMap methods are used to verify the design affordance of the designed mockups of the Smart TV UIs. The GazeTrail shows the subject's ocular scan path by drawing a connected path of the recorded gaze position data on the Smart TV UI mockup design. LookZone calculates the area of interest in certain a Smart TV UI . HeatMap shows the intensity calculated from the GazePoint results. Data that collected on fixations and saccades from an eye-tracker will be visualized in an eye tracking software as gaze plot and heat map. The information that was collected from eye tracking evaluation is then provided for the design affordance analysis. 


\section{ANALYSIS OF USER INTERFACE FEATURESAND DESIGN MOCKUP}

\section{A. Results of QFD-AHP Model Analysis}

Figure 1 shows the QFD matrix results. Based on QFD matrix analysis. the smart interactive user interface and privacy settings are two of the most important features of Smart TVs, followed by gesture and voice control, customization of personal settings, and layout adaptation. These visualized results show that the UI design is very important to Smart TVs.

In comparison with technical features, gesture recognition and facial recognition are highly prized by respondents. Privacy via encryption and decryption, and traditional/single sign-in on account management are also required by customers. Respondents agreed that Apple TVs and Smart TVs have user-friendly interfaces. The privacy feature has already been developed by Apple TV, general Smart TV, and Google TV. The QFD matrix results comprehensively show a significant role to help the development. These results are also evaluated and calculated via the AHP. Each criterion is compared to another, such that the importance weight is derived.

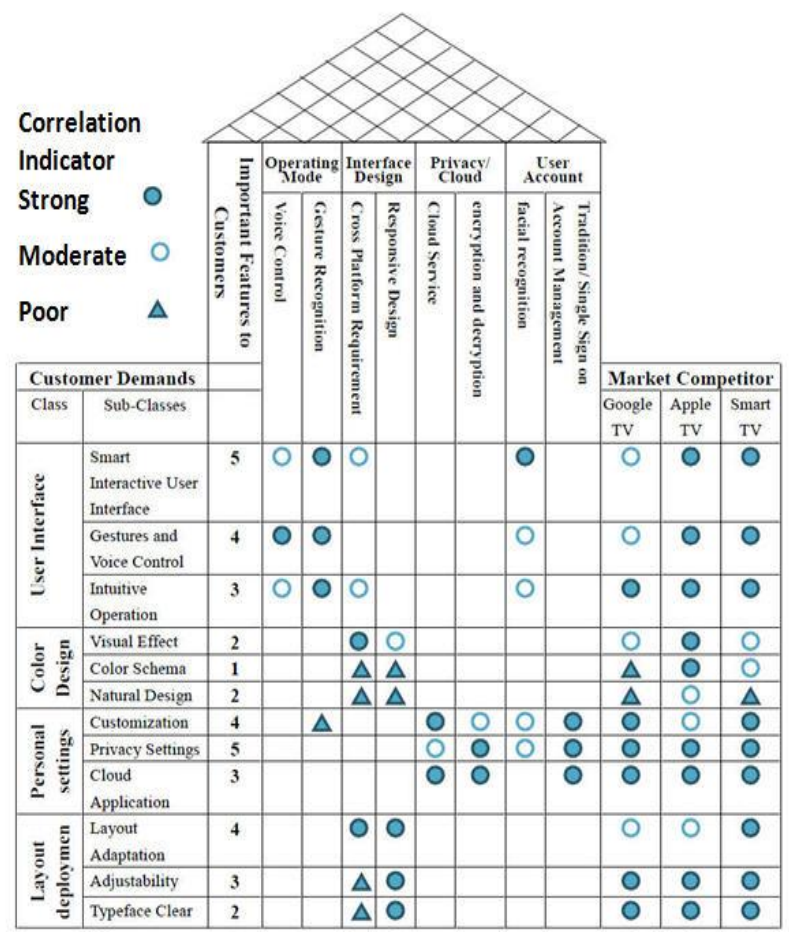

Fig. 1. Quality Function Deployment Matrix Results

Features in the QFD matrix are further processed as criteria in a questionnaire. To collect pair wise comparison results, 30 questionnaires were dispatched to inter-disciplinary experts, including faculty, researchers, and professionals in the fields of computer science, electronic engineering, and interaction design.

The final results of QFD-AHP model are presented in Table 1. There is a correlation exists between the QFD matrix weight scale and the weights and ranking from the AHP. The most demanded feature of a Smart TV HCI is the user interface (31\%), which is followed by a visual design (27\%). Demand for the personal settings (22\%) differs little from that for layout design (20\%). However, the top five design priorities for Smart TV HCI and UI are layout adaptation (46\%), a smart interactive user interface (39\%), personal customization(39\%), natural visual design (36\%) and intuitive operation of the user interface $(35 \%)$. The top three features derived from the AHP are similar to those specified by the QFD weights? However, the design features with the fourth and fifth priorities are very different from those obtained using the OFD. Gesture and voice control has a high priority in the QFD matrix, but a low weight by the AHP method, probably because this customer requirement. Additional efforts must be made to provide gesture and voice control when designing multimodal interaction for Smart TVs. The results provide some guidelines for industry in the design of Smart TV HCIs and UIs.

TABLE I. THE QFD AND AHP RESULTS

\begin{tabular}{|c|c|c|c|c|}
\hline Class & $\begin{array}{l}\text { Smart TV HCI } \\
\text { Design } \\
\text { Features }\end{array}$ & $\begin{array}{l}\text { QFD } \\
\text { Weights }\end{array}$ & $\begin{array}{l}\text { AHP } \\
\text { Weights }\end{array}$ & $\begin{array}{l}\text { AHP } \\
\text { Overall } \\
\text { Ranking }\end{array}$ \\
\hline \multirow{3}{*}{$\begin{array}{l}\text { User } \\
\text { Interface } \\
(\mathbf{3 1 \%})\end{array}$} & $\begin{array}{l}\text { Smart } \\
\text { Interactive User } \\
\text { Interface }\end{array}$ & 5 & $39 \%$ & 2 \\
\hline & $\begin{array}{l}\text { Gestures and } \\
\text { Voice Control }\end{array}$ & 4 & $26 \%$ & 11 \\
\hline & $\begin{array}{l}\text { Intuitive } \\
\text { operation }\end{array}$ & 3 & $35 \%$ & 5 \\
\hline \multirow{3}{*}{$\begin{array}{l}\text { Visual } \\
\text { Design } \\
(27 \%)\end{array}$} & Visual Effect & 2 & $31 \%$ & 8 \\
\hline & $\begin{array}{l}\text { Color } \\
\text { Brightness }\end{array}$ & 1 & $33 \%$ & 6 \\
\hline & Natural Design & 2 & $36 \%$ & 4 \\
\hline \multirow{3}{*}{$\begin{array}{l}\text { Personal } \\
\text { Settings } \\
(22 \%)\end{array}$} & Customization & 5 & $39 \%$ & 2 \\
\hline & Privacy Settings & 4 & $32 \%$ & 7 \\
\hline & $\begin{array}{l}\text { Cloud } \\
\text { Application }\end{array}$ & 3 & $29 \%$ & 9 \\
\hline \multirow{3}{*}{$\begin{array}{l}\text { Layout } \\
\text { Deploym } \\
\text { ent } \\
(20 \%)\end{array}$} & $\begin{array}{l}\text { Adaptive } \\
\text { Layout }\end{array}$ & 4 & $46 \%$ & 1 \\
\hline & Clarity & 3 & $28 \%$ & 10 \\
\hline & Clear typeface & 2 & $26 \%$ & 11 \\
\hline
\end{tabular}

\section{B. Mockups Smart TV User Interface Designs}

Figures 2 to 4 below present mockup designs of Smart TV UIs. The basic requirement of the design of these new interfaces is that users can quickly gain an understanding of which elements on the screen can be used to navigate. Users often spend only a few seconds to familiarize themselves with all of the elements of the interface. They should be able to establish a mental floor plan of the interface. Elements that are the most visually prominent will receive the most attention and will help to shape the user's perception of the interface. Accordingly, visual affordance will provide a cue to users that a certain element is clickable. These mockups will be used in the eye tracking evaluation. 


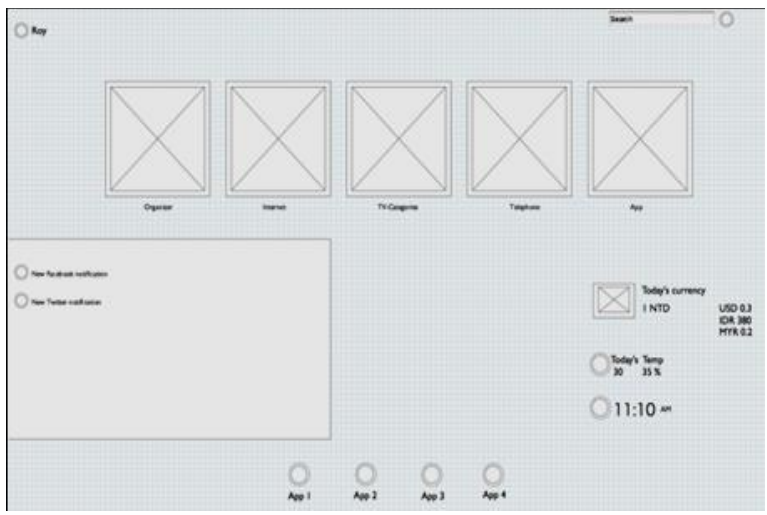

Fig. 2. Smart TV UI Design Mockup 1

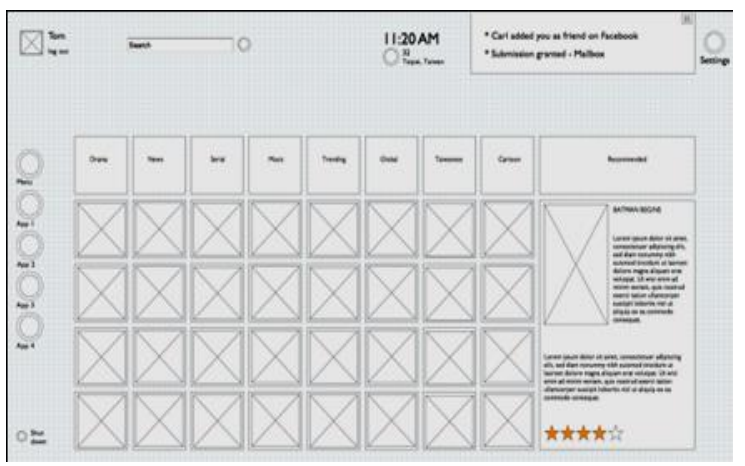

Fig. 3. Smart TV UI Mockup 2

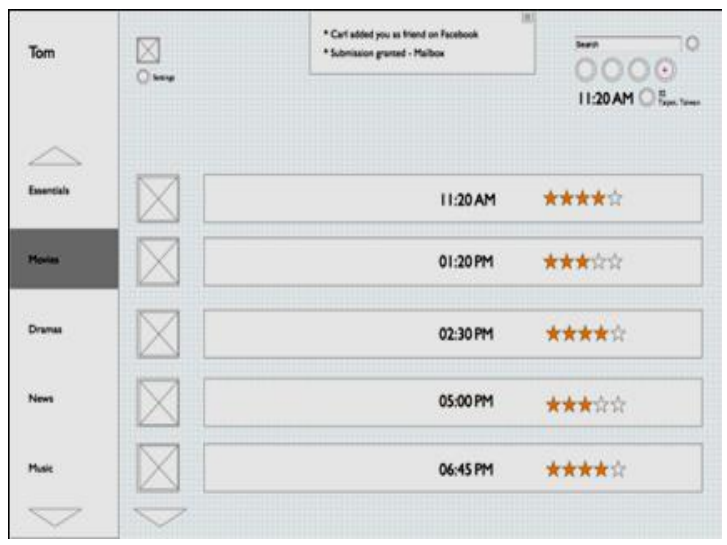

Fig. 4. Smart TV UI Mockup 3

\section{Design of Eye-Tracking Evaluation}

Before the experiment was begun, five look zones (LZ) were established on the interface in different colors to help to track each participant's eye movement. These five LZs correspond to Search, Notification, Launcher, User Account, and Movie (Categories). The pictures below show the locations of the LZs on the screen. The time and position of viewing are recorded to determine gaze time and the sequence of eye path.

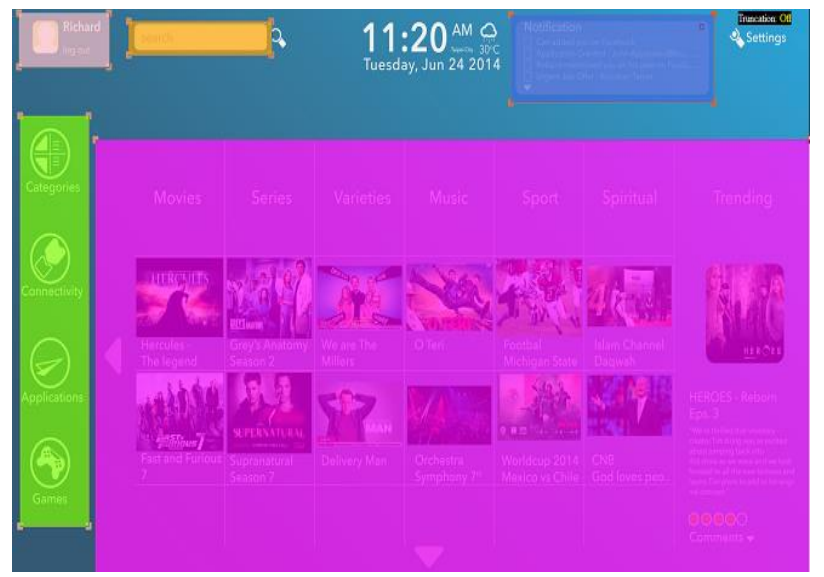

Fig. 5. Look Zones on Screen

\section{Evaluation AND DisCUSSION}

A. Analysis of Efficiency of Mockup of Design of Smart TV UI

Eye tracking experiment was carried out using the Gaze Tracker EyeLink 1000 Plus. Six participants took the test. Participant were asked to sync their eye's gaze to the sensor before beginning the task. Participants were required to identify the contents of a selected Smart TV UI design, while the Gaze Tracker recorded their eye position and searching time. The obtained were presented as eye movement points and timed trajectories.

The following tables presents the time taken by each participant while searching and identifying the contents of each Smart TV UIs mockup design.

TABLE II. GAZE TIME ReCORd OF PARTICIPANT 01

\begin{tabular}{|l|l|l|l|}
\hline Contents & UI \#1 & UI \#2 & UI \#3 \\
\hline Categories & 3.63 & 3.7 & 1.8 \\
\hline Search (sec) & 2.05 & 3.85 & 1.5 \\
\hline User Account(sec) & 3.31 & 1.15 & 3.53 \\
\hline Movie Selection (sec) & 3.76 & 4.98 & 1.66 \\
\hline App Launcher (sec) & 3.06 & 3.03 & 3.45 \\
\hline Notification (sec) & 2.18 & 5.43 & 3.43 \\
\hline Average Time (sec) & 3 & 3.7 & 2.6 \\
\hline
\end{tabular}

TABLE III. TIME RECORD OF PARTICIPANT 02

\begin{tabular}{|l|l|l|l|}
\hline Contents & UI \#1 & UI \#2 & UI \#3 \\
\hline Categories (sec) & 5.03 & 3.3 & 1.48 \\
\hline Search (sec) & 1.71 & 1.06 & 5.48 \\
\hline Users (sec) & 3.18 & 2.21 & 1.88 \\
\hline Movie Selection (sec) & 4.28 & 2.13 & 1.3 \\
\hline App Launcher (sec) & 3.15 & 2.48 & 4.08 \\
\hline Notification (sec) & 1.53 & 1.54 & 2 \\
\hline Average Time (sec) & 3.1 & 2.1 & 2.7 \\
\hline
\end{tabular}


TABLE IV. TIME RECORD OF PARTICIPANT 03

\begin{tabular}{|l|l|l|l|}
\hline Contents & UI \#1 & UI \#2 & UI \#3 \\
\hline Categories (sec) & 2.11 & 4.66 & 1.33 \\
\hline Search (sec) & 1.93 & 2.33 & 8.5 \\
\hline Users Account(sec) & 2.6 & 4.88 & 4.2 \\
\hline Movie Selection (sec) & 3.18 & 4.46 & 2.55 \\
\hline App Launcher (sec) & 5.65 & 2.75 & 1.78 \\
\hline Notification (sec) & 4.05 & 3.63 & 5.23 \\
\hline Average Time (sec) & 3.3 & 3.8 & 3.9 \\
\hline
\end{tabular}

TABLE V. TIME RECORD OF PARTICIPANT 04

\begin{tabular}{|l|l|l|l|}
\hline Contents & UI \#1 & UI \#2 & UI \#3 \\
\hline Categories (sec) & 2.56 & 3.11 & 2.66 \\
\hline Search (sec) & 1.76 & 2.96 & 2.35 \\
\hline Users Account(sec) & 2.33 & 2.16 & 1.55 \\
\hline Movie Selection (sec) & 11.58 & 3.33 & 1.55 \\
\hline App Launcher (sec) & 6.41 & 1.95 & 16.7 \\
\hline Notification (sec) & 1.23 & 2.53 & 2.1 \\
\hline Average Time (sec) & 4.3 & 2.7 & 4.5 \\
\hline
\end{tabular}

TABLE VI. TIME RECORD OF PARTICIPANT 05

\begin{tabular}{|l|l|l|l|}
\hline Contents & UI \#1 & UI \#2 & UI \#3 \\
\hline Categories (sec) & 8.68 & 2.36 & 6.11 \\
\hline Search (sec) & 2.91 & 1.26 & 1.81 \\
\hline Users Account(sec) & 1.13 & 1.38 & 1.98 \\
\hline Movie (sec) Selection & 13.33 & 2.76 & 3.2 \\
\hline App Launcher (sec) & 2.58 & 1.28 & 6.6 \\
\hline Notification (sec) & 4.43 & 3.16 & 7.85 \\
\hline Average Time (sec) & 5.5 & 2 & 4.6 \\
\hline
\end{tabular}

TABLE VII. TIME RECORD OF PARTICIPANT 06

\begin{tabular}{|l|l|l|l|}
\hline Contents & UI \#1 & UI \#2 & UI \#3 \\
\hline Categories (sec) & 3.08 & 5 & 14.06 \\
\hline Search (sec) & 2.03 & 2.76 & 2.56 \\
\hline Users Account (sec) & 2.21 & 2.4 & 2 \\
\hline Movie (sec) Selection & 4.51 & 3.96 & 3.93 \\
\hline App Launcher (sec) & 1.68 & 2.98 & 6.3 \\
\hline Notification (sec) & 5.46 & 3.3 & 2.58 \\
\hline Average Time (sec) & 3.1 & 3.4 & 5.2 \\
\hline
\end{tabular}

Table 8 presents the average of the times taken to complete identify the contents of each Smart TV UIs design mockup.

TABLE VIII. GRAND AVERAGE TIME FOR PARTICIPANT TO COMPLETE IDENTIFY THE CONTENTS OF EACH SMART TV UI DESIGN

\begin{tabular}{|l|l|l|l|}
\hline & UI \#1 & UI \#2 & UI \#3 \\
\hline Participate 01 & 3 & 3.7 & 2.6 \\
\hline Participate 02 & 3.1 & 2.1 & 2.7 \\
\hline Participate 03 & 3.2 & 3.8 & 3.9 \\
\hline Participate 04 & 4.3 & 2.7 & 4.5 \\
\hline Participate 05 & 5.5 & 2 & 4.6 \\
\hline Participate 06 & 3.1 & 3.4 & 5.2 \\
\hline Average Time & 3.7 & 3.0 & 3.9 \\
\hline
\end{tabular}

This table demonstrates that the users can perceive the proposed Smart TV UI mockup design less than 5 s, revealing the efficiency of the design. Efficiency can be described as the speed and accuracy with which users can complete tasks for which they use the UIs. The results also reveal that the design can create a favorable user experience of the Smart TV UIs as it guides the participants effortlessly through the UIs to reach their goal.
All of the participants in this research had little trouble in identifying the contents in the test, indicating that the mockups of the Smart TV UI designs that are presented in this research are sufficiently effective. Effectiveness is the completeness and accuracy with which users achieve specified goals; it is determined by determined whether the user's goals are met successfully and whether all work is correct. Effectiveness is the driving force behind successful task completion and helps users to complete their goals. The effectiveness of these Smart TV UIs is determined by whether users can locate and use the navigation option to take them to the expected location. UI\#2 performs best, followed by UI\#1. Therefore, in the next part, UI \#2 and UI\#1 are used as the wireframes for the evaluation of eye tracking.

\section{B. Eye Tracking: Evaluation and Discussion}

Five look zones (LZ) were established to determine the eye movement of each participant. These five LZ are set for Search, Notification, Launcher, and Movie (Categories). The following figures show the locations of the set-up LZs on the screen. In Fig. 6, the pink rectangle represents the User LZ; the yellow rectangle represents the Search LZ; the blue rectangle represents the Notification LZ; the green rectangle represents the Launcher LZ, and the purple rectangle represents the Movie LZ. Each table following a figure presents detailed information about eye movement on a slide of the mockup design of the Smart TV UIs and the five look zones. UI\#2 and UI\#1 are installed on the eye-tracking device and six participants are involved in the evaluation. The system recorded tracking data from only three participants owing to some problems with the system. Therefore, only three datasets are analyzed.

The eye tracking results demonstrate that LookZone-Movie Selection receives more gazing time than others(total time in zone: $84.37 \mathrm{~s}$ for three participants), and LookZone-User is also perceived as important (gaze point count: 3157 times for three participants). The grid system design helps these three participants to distinguish parts of interface, and all participants described the process of movie selection systematically. LookZone-User Account and LookZone-Launcher are located in the same column, so the three participants all found moving between these two sections convenient.

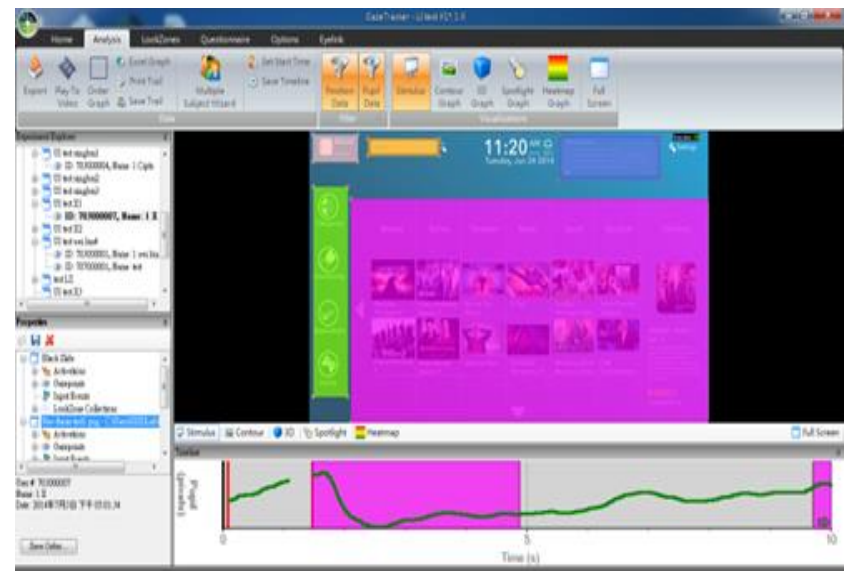

Fig. 6. LookZones for Smart TV UI\#2 


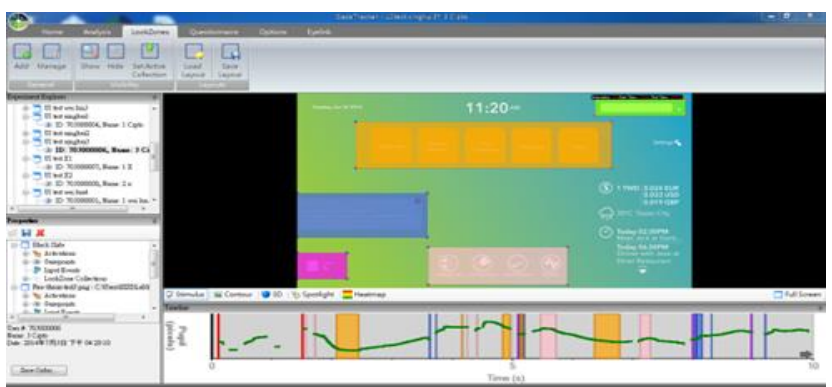

Fig. 7. LookZones for UI\#1

TABLE IX. GAZE Tracker RESUlts FOR UI\#2

\begin{tabular}{|l|l|l|l|}
\hline UI \#2 & P07 & P08 & P09 \\
\hline Slide Metrics & & & \\
\hline Total time shown (seconds) & 93 & 79 & 74 \\
\hline Total time tracked (seconds) & 21 & 77 & 63 \\
\hline LookZone - Search & & & \\
\hline Number of times zone observed & 0 & 1 & 15 \\
\hline Gazepoint count & 0 & 5 & 427 \\
\hline Gazepoint count / Total time in zone & 0 & 625 & 430 \\
\hline LookZone - Launcher & & & \\
\hline Number of times zone observed & 81 & 8 & 15 \\
\hline Gazepoint count & 2056 & 431 & 414 \\
\hline Gazepoint count / Total time in zone & 420 & 508 & 487 \\
\hline LookZone - Notification & & & \\
\hline Number of times zone observed & 1 & 2 & 15 \\
\hline Gazepoint count & 1 & 11 & 1297 \\
\hline Gazepoint count / Total time in zone & 0 & 611 & 405 \\
\hline LookZone - User & & & \\
\hline Number of times zone observed & 6 & 8 & 8 \\
\hline Gazepoint count & 20 & 2079 & 1058 \\
\hline Gazepoint count / Total time in zone & 741 & 490 & 391 \\
\hline LookZone - Movie Selection & & & \\
\hline Number of times zone observed & 313 & 58 & 77 \\
\hline Gazepoint count & 1814 & 24286 & 12303 \\
\hline Gazepoint count / Total time in zone & 529 & 483 & 402 \\
\hline
\end{tabular}

TABLE X. GaZE Tracker Results For UI\#1

\begin{tabular}{|l|l|l|l|}
\hline UI \#1 & P07 & P08 & P09 \\
\hline Slide Metrics & & & \\
\hline Total time shown (seconds) & 78 & 48 & 80 \\
\hline Total time tracked (seconds) & 33 & 47 & 55 \\
\hline LookZone - Search & & & \\
\hline Number of times zone observed & 4 & 4 & 7 \\
\hline Gazepoint count & 4 & 8 & 317 \\
\hline Gazepoint count / Total time in zone & 0 & 1143 & 418 \\
\hline LookZone - Launcher & & & \\
\hline Number of times zone observed & 16 & 13 & 52 \\
\hline Gazepoint count & 798 & 2525 & 2432 \\
\hline Gazepoint count / Total time in zone & 407 & 401 & 407 \\
\hline LookZone - Notification & & & \\
\hline Number of times zone observed & 47 & 15 & 40 \\
\hline Gazepoint count & 676 & 2678 & 450 \\
\hline Gazepoint count / Total time in zone & 425 & 397 & 174 \\
\hline LookZone - Users & & & \\
\hline Number of times zone observed & 2 & 4 & 13 \\
\hline Gazepoint count & 117 & 8 & 173 \\
\hline
\end{tabular}

\begin{tabular}{|l|l|l|l|} 
Gazepoint count / Total time in zone & 402 & 1333 & 434 \\
\hline LookZone - Categories & & & \\
\hline Number of times zone observed & 98 & 8 & 38 \\
\hline Gazepoint count & 4807 & 2525 & 4549 \\
\hline Gazepoint count / Total time in zone & 408 & 401 & 398 \\
\hline
\end{tabular}

As presented in Table 10, in UI\#1, look zones "categories" is not one of the specified LZs categories and launcher attract more gazes than the other zones, but many fewer than all look zones in UI\#2. "Gaze Point Count" is the number of times an eye hits a defined look zone. UI\#1 receives more gazes than UI\#2. "LookZone-User Account" receives the fewest gazes on UI\#1. However, on UI\#2, "LookZone-Search" and "LookZone-Notification" receive fewest gazes, while "LookZone-User Account" receives many gazes .In Figs. 7 and 8 , the heat map shows the gazing time and number of gazes by all participant. A comparison of the eye-tracking results obtained using QFD and AHP reveals that layout adaptation and Smart interactive user interface features can make the interface more effective and enjoyable to use.

As revealed by the results, the grid system as designed by the creation of digital mockups, helped participants to see separations among the contents of a UI. This method also helps participants to appreciate what a UI provides and where important contents are located on the screen. A wireframe mockup is affordable and easy to create and provides a way to see how users actually interact with the Smart TV UI designs that were proposed in this research. Since the workshop herein was based on "design thinking", the processes of observing, and approaching people through visual work helped to yield the desired results. Since this research is a response to a previous workshop with multidisciplinary master students, the QFD matrix and AHP results can be used as references for the design process. Time of completion is crucial in this research; the speed with which a person identify the UI contents is related to the ease with which they perceived the corresponding visual aid, and therefore to affordance.

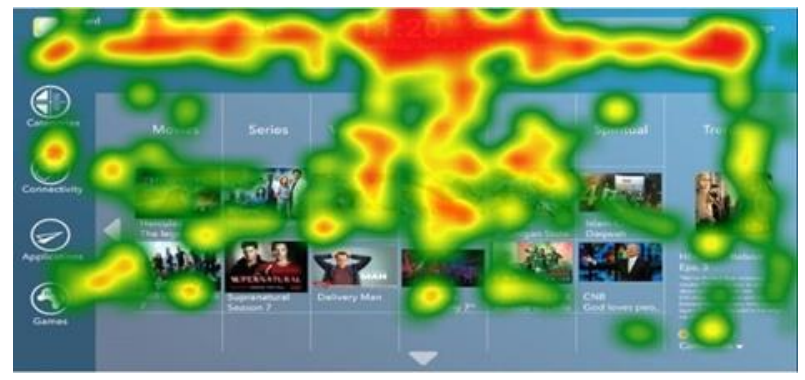

Fig. 8. Heat map for Smart TV UI\#2

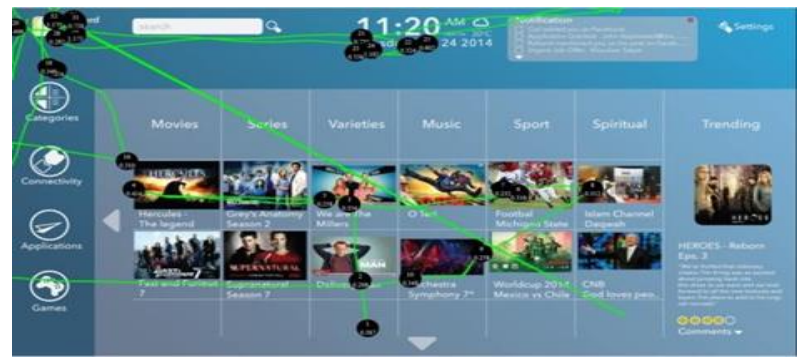

Fig. 9. Gaze point(s: check) for Smart TV UI\#2 
Research has established that users of any new interface need to quickly gain an understanding of which elements on the screen can be used. Users frequently take only a few seconds to familiarize themselves with all of the elements on the page and then establish a mental plan of the interface in a very short time. Therefore, design blocks or elements that are the most visually prominent attract the most attention and will help to shape a user's perception of the interface. The information in Tables 9 and 10 indicates the visual affordance of the Smart TV UI mockup designs that are proposed in this research. The overall results provide clues to users that certain elements are operable Further analysis based on the method with Gestalt principles, which are time-tested methods that shape the visual hierarchy that a user will see, will be conducted in the future to revise the original mockup Smart TV UIs.

\section{CONCLUSIONS AND FUTURE WORKS}

Creating a usable user interface for a Smart TV is critical to a positive user experience. Designing interactive content would have been difficult without a pertinent method. Indeed, in the field of design, people are encouraged to create their own solutions to problems, but appropriate methods are highly recommended to be considered before any design is conducted, to facilitate the effective solution to particular problems.

This research aims to proposed a comprehensive process for designing and evaluation Smart TV UIs with high affordance. A design process is implemented, based on the output of design thinking, and the results are evaluated and analyzed Also, interdisciplinary collaboration among people from various fields and backgrounds were engaged to ensure that the proper design approaches were taken. QFD and AHP supported the initial process of creating a new prototype specification that accounted users' desired features and correlating to the possibilities of engineering technologies. This scheme comprehends incorporates users' experiences and allows problems with this system to be identified and the system to be assessed. Eye tracking verifies the effectiveness and efficiency of the Smart TV UIs mockup design by recording of the gaze of users. This technology provides clear traces and calculates the numbers, percentages, and time of a user's eye movements.

In conclusion, the iterative process of redesigning Smart TV UIs that is proposed in this research may help to improve its effectiveness and efficiency, and enable changes in users' behaviors and needs to be responded to. Visual affordance provides clues to users that some elements are operable. Further analysis based on the methods with Gestalt principles, which are time-tested methods that shape the visual hierarchy that a user will see, will be performed in future research to revise the original Smart TV UI mockup designs. Moreover, in future work, many possible interactive designs will be developed. Voice and gesture-based interactions or other affordable inputs may elevate the user's experience of communicating through the interface, possibly opening up another basis for a universal design that would enable a disabled person to interact with the user interface. Even though many possible types of input connectivity may exist, a user's behavior should be given a high priority in the design process.

\section{ACKNOWLEDGMENTS}

This work was supported the Ministry of Science and Technology, Taiwan, under the projects NSC 101-2219-E-027-007 and MOST 103-2221-E-027-062. The assistance of my students Cheih-Ju Huang and Cipto Hartanto is greatly appreciated. Ted Knoy is appreciated for his editorial assistance.

\section{REFERENCES}

[1] K. Merkel, "Hybrid broadcast broadband TV, the new way to a comprehensive TV experience," in Electronic Media Technology (CEMT), 2011 14th ITG Conference on, 2011, pp. 1-4.

[2] L. B. Yuste and S. Al-Majeed, "Effective synchronisation of hybrid broadcast and broadband TV," in 2012 IEEE International Conference on Consumer Electronics (ICCE), Las Vegas, NV, USA, 2012, pp. $160-161$.

[3] S.-M. Wang, "Service Design for Developing Multimodal Human Computer Interaction for Smart TVs," International Journal of Advanced Computer Science and Applications, vol. 6, pp. 227-234, 2015.

[4] P. Hamisu, G. Heinrich, C. Jung, V. Hahn, C. Duarte, P. Langdon, and P. Biswas, "Accessible UI design and multimodal interaction through hybrid TV platforms: towards a virtual-user centered design framework," in Universal Access in Human-Computer Interaction. Users Diversity, ed: Springer, 2011, pp. 32-41.

[5] S.-M. Wang, V. Bailey, and C. Hartanto, "Developing Hybrid Internet Broadcast Television user interface base on user experience design principle," in Consumer Electronics-Taiwan (ICCE-TW), 2014 IEEE International Conference on, Taipei, Taiwan, 2014, pp. 61-62.

[6] O. Martinez-Bonastre, M.-J. Montpetit, and P. Cesar, "IP-based tv technologies, services, and multidisciplinary applications," Communications Magazine, IEEE, vol. 51, 2013.

[7] S.-M. Wang and C. J. Huang, "QFD and AHP integration for Smart TV human-computer interaction design," in Consumer Electronics-Taiwan (ICCE-TW), 2014 IEEE International Conference on, Taipei, Taiwan, 2014, pp. 59-60.

[8] T.-H. You, Y.-J. Wu, C.-L. Lin, and Y. Chuang, "Enhancing People's Television Experience by Capturing, Memoing, Sharing, and Mixing," in Cross-Cultural Design. Methods, Practice, and Case Studies, ed: Springer, 2013, pp. 510-518.

[9] A. Schall, "Eye tracking insights into effective navigation design," in Design, User Experience, and Usability. Theories, Methods, and Tools for Designing the User Experience, ed: Springer, 2014, pp. 363-370.

[10] Z. Obrenovic and D. Starcevic, "Modeling multimodal human-computer interaction," Computer, vol. 37, pp. 65-72, 2004.

[11] B. Moggridge and B. Atkinson, Designing interactions vol. 14: MIT press Cambridge, 2007.

[12] M. Tambe, "TV Human Interface: Different Paradigm from that of PC and Mobile," IEEE Code of Ethics, 2012.

[13] S.-F. M. Liang, Y.-C. Kuo, and S.-C. Chen, "Identifying Usability Problems in a Smart TV Music Service," in Cross-Cultural Design. Methods, Practice, and Case Studies, ed: Springer, 2013, pp. 306-312.

[14] V. Vinayagamoorthy, P. Allen, M. Hammond, and M. Evans, "Researching the user experience for connected tv: a case study," in CHI'12 Extended Abstracts on Human Factors in Computing Systems, 2012, pp. 589-604.

[15] Y. Lu, Y. Kim, X. Y. Dou, and S. Kumar, "Promote physical activity among college students: Using media richness and interactivity in web design," Computers in Human Behavior, vol. 41, pp. 40-50, 2014.

[16] S. Abrahão, E. Insfran, and A. Fernandez, "Designing Highly Usable Web Applications," 2014.

[17] T. Ha and S. Lee, "User behavior model based on affordances and emotions: A new approach for an optimal use method in product-user interactions," International Journal of Human-Computer Interaction, 2014.

[18] A. L. Blackler, V. Popovic, and D. P. Mahar, "Applying and testing design for intuitive interaction," International Journal of Design Sciences and Technology, vol. 20, pp. 7-26, 2014. 
[19] P. Cormier, A. Olewnik, and K. Lewis, "Toward a formalization of affordance modeling for engineering design," Research in Engineering Design, vol. 25, pp. 259-277, 2014.

[20] E. J. Park, "Creating a sustainable business model for the TV industry: strategic analysis on the Smart TV ecosystem," Master, Sloan School of Management, Massachusetts Institute of Technology, 2014.

[21] P. Ramakrisnan, A. Jaafar, F. H. A. Razak, and D. A. Ramba, "Evaluation of user interface design for leaning management system (LMS): investigating student's eye tracking pattern and experiences," Procedia-Social and Behavioral Sciences, vol. 67, pp. 527-537, 2012.

[22] S. Moritz, "Service design: practical access to an evolving field," Cologne, Germany: Köln International School of Design, 2005.

[23] T. L. Saaty, "An exposition of the AHP in reply to the paper "remarks on the analytic hierarchy process"," Management science, vol. 36, pp. 259-268, 1990.

[24] T. L. Saaty, "Decision making with the analytic hierarchy process," International journal of services sciences, vol. 1, pp. 83-98, 2008.

[25] F. De Felice and A. Petrillo, "A multiple choice decision analysis: an integrated QFD-AHP model for the assessment of customer needs," International Journal of Engineering, Science and Technology, vol. 2, 2011.

[26] J. J. Gibson, "The theory of affordances," in Perceiving, Acting, and Knowing: toward an ecological psychology, R. E. Shaw and J. Bransford, Eds., ed Hillsdale, NJ: Lawrence Erlbaum Associates, 1977, pp. $67-82$.
[27] D. A. Norman, "Affordance, conventions, and design," interactions, vol. 6, pp. 38-43, 1999.

[28] D. Bruneau, M. A. Sasse, and J. McCarthy, "The eyes never lie: The use of eye tracking data in HCI research," in Proceedings of the CHI, 2002, p. 25.

[29] S. Djamasbi, M. Siegel, and T. Tullis, "Generation Y, web design, and eye tracking," International journal of human-computer studies, vol. 68, pp. 307-323, 2010.

[30] L. E. Nacke, M. N. Grimshaw, and C. A. Lindley, "More than a feeling: Measurement of sonic user experience and psychophysiology in a first-person shooter game," Interacting with Computers, vol. 22, pp. 336-343, 2010.

[31] S. P. Roth, A. N. Tuch, E. D. Mekler, J. A. Bargas-Avila, and K. Opwis, "Location matters, especially for non-salient features-An eye-tracking study on the effects of web object placement on different types of websites," International journal of human-computer studies, vol. 71, pp. 228-235, 2013.

[32] K. D. Goepel, "Implementing the analytic hierarchy process as a standard method for multi-criteria decision making in corporate enterprises-a new AHP excel template with multiple inputs," in Proceedings of the international symposium on the analytic hierarchy process, Kuala Lumpur, Malaysia, 2013. 